\title{
The additive role of diffusion-weighted magnetic resonance imaging to axillary nodal status evaluation in cases of newly diagnosed breast cancer
}

Dalia Salaheldin Elmesidy ${ }^{1,2^{*}}$ (D), Eman Ahmed Mohammed Omar Badawy ${ }^{1}$, Rasha Mohammed Kamal ${ }^{1,2}$, Emad Salah Eldin Khallaf ${ }^{3}$ and Rasha Wessam AbdelRahman ${ }^{1,2}$

\begin{abstract}
Background: Axillary nodal status is crucial for the management of cases with recently diagnosed breast cancer; usually addressed via axillary ultrasonography (US) along with tissue sampling in case of suspicion. Axillary nodal dissection and sentinel biopsy may be done, but are rather invasive, carrying a potential complication risk, which raises the need for non-invasive, reliable, pre-operative axillary imaging. We aimed at evaluating the performance of diffusion-weighted imaging (DWI) regarding preoperative axillary evaluation, using functional information derived from diffusion capacity differences between benign and malignant tissue. We included 77 axillary nodes from 77 patients (age range 20-78 years, mean $50 \pm 12.6$ SD) in our prospective study, presenting with variable clinical breast complaints, all scoring BIRADS 4/5 on sonomammography (SM). They underwent axillary evaluation by both US and DW-MRI where US classified nodes into benign, indeterminate, or malignant by evaluating nodal size, shape, cortical thickness, and hilar fat. Qualitative DWI classified them into either restricted or not and a cut-off apparent diffusion coefficient (ADC) value was calculated to differentiate benign and malignant nodal involvement. Results for each modality were correlated to those of final histopathology, which served as the standard of reference.

Results: The calculated sensitivity, specificity, accuracy, PPV, and NPV for US was 100\%, 36.6\%, 75.3\%, 71.2\%, and $100 \%$, respectively. Statistical indices for qualitative DWI were $76.6 \%, 63.3 \%, 76.6 \%, 63.3 \%$, and $71.4 \%$, respectively $(P$ value $<0.001)$. The calculated cut off value for ADC between infiltrated and non-infiltrated nodes was $0.95 \times 10^{-3}$ $\mathrm{mm}^{2} / \mathrm{s}$ concluding statistical indices of $76.6 \%, 63.3 \%, 76.6 \%, 63.3 \%$, and $71.4 \%$, respectively ( $P$ value $\left.<0.001\right)$.

Conclusion: Combining DW-MRI to conventional US improves diagnostic specificity and overall accuracy of preoperative axillary evaluation of patients with recently discovered breast cancer.
\end{abstract}

Keywords: Axillary nodes, Diffusion-weighted imaging, Breast cancer

\footnotetext{
* Correspondence: daliamsd@windowslive.com

'Department of Diagnostic and Interventional Radiology, Faculty of

Medicine, Cairo University, Cairo, Egypt

${ }^{2}$ Department of Diagnostic and Interventional Radiology, Baheya Foundation,

Cairo, Egypt

Full list of author information is available at the end of the article
}

\section{Springer Open}

(-) The Author(s). 2021 Open Access This article is licensed under a Creative Commons Attribution 4.0 International License, which permits use, sharing, adaptation, distribution and reproduction in any medium or format, as long as you give appropriate credit to the original author(s) and the source, provide a link to the Creative Commons licence, and indicate if changes were made. The images or other third party material in this article are included in the article's Creative Commons licence, unless indicated otherwise in a credit line to the material. If material is not included in the article's Creative Commons licence and your intended use is not permitted by statutory regulation or exceeds the permitted use, you will need to obtain permission directly from the copyright holder. To view a copy of this licence, visit http://creativecommons.org/licenses/by/4.0/. 


\section{Background}

Axillary nodal status is a cornerstone for cases of recently diagnosed breast cancer which is commonly evaluated by axillary sonography (US) along with sampling when suspicious [1]. It has been shown that complete nodal axillary dissection (ALND) for cases with limited nodal axillary involvement (1-3 nodes) is not useful [2]. Rather invasive techniques (such as nodal biopsy) carry a potential risk of complications and so a need for noninvasive axillary assessment has emerged [3].

Diffusion-weighted magnetic resonance imaging (DWMRI) utilizes water molecular mobility (Brownian motion) to identify differences in the capacity of diffusion (and consequently signal differences) between malignant and benign tissues providing both functional and morphological data, where water molecule diffusivity and apparent diffusion coefficient (ADC) are markedly affected by lesion cellularity [4].

The sensitivity of diffusion is affected by altering the $b$ value (gradient strength for diffusion) which is directly proportional to the amplitude and duration of the gradient, as well as the interval of time between gradients (measured in $\mathrm{s} / \mathrm{mm}^{2}$ ). Free water molecules (high motion degree) will have lower signal at a lower $b$ value $(b$ $=50-100 \mathrm{~s} / \mathrm{mm}^{2}$ ) (perfusion) while slower moving water commonly need higher $b$ values $\left(b=800 \mathrm{~s} / \mathrm{mm}^{2}\right)$ [5]

\section{Aim of work}

We aimed at evaluating the added role of DW-MRI (to that of US) for diagnosis and accurate staging of the axillary nodal status in patients with recently diagnosed breast cancer, being a preoperative non-invasive tool.

\section{Methods}

In our prospective study, we included 77 axillary nodes in 77 female patients who presented to our institution during the period from February 2017 to April 2019 (age range 20-78 years, mean $50 \pm 12.6 \mathrm{SD}$ ) who had suspicious lesions on sonomammography (SM) (scoring BIRADS 4/5). These patients presented to the General Surgery Department at our institution with variable complaints including inflammatory breast changes, breast or axillary lumps. We excluded patients scoring BIRADS 1, 2, or 3 on SM or those with general contraindications to magnetic resonance imaging (MRI) (such as pacemakers or cochlear implants). We also excluded those who had previous ALND, or those who received any treatment that may affect the outcome of the study. All patients provided informed consent and our institutional ethical approval was obtained. The most suspicious axillary lymph node on the same side of the primary breast lesion was included in the study. Criteria for the most suspicious lymph node were focal or marked cortical thickening, hilar fat loss, or decreased longitudinal to transverse dimension ratio ( $\mathrm{L} / \mathrm{T}$ ratio). If no suspicious nodes were found, we included the largest one in the study.

Patients underwent detailed history taking (including name, age, marital status, residence, history of previous illness, etc.), clinical breast examination, SM, and USguided biopsy from any suspicious lesions, as well as breast dynamic DW-MRI.

For US, the study was conducted using a General Electric Medical Systems LOGIQ S8 or LOGIQ P US device that includes a multi-frequency linear probe operating at 7.5 to $12 \mathrm{MHz}$. Patients were examined in the classic oblique supine position with their ipsilateral arm abducted and externally rotated so that their hand is behind their head. The nodes of concern were focused upon where the protocol of scanning included real-time longitudinal and transverse imaging. Each node was examined for size (long and short axis in centimeters), shape (as indicated by its $\mathrm{L} / \mathrm{T}$ ratio which if more than 2 , the node is considered ovoid while if less than 2 , it is considered non-ovoid), cortical thickening (if present whether it is diffuse or focal), and hilar fat (preserved or not). If more than one node was found in the same axilla, the most suspicious was analyzed. The most suspicious node on the ipsilateral side of the primary lesion was included in the study. Axillary nodes chosen as most suspicious to be included in the study were those having one or more of the following criteria on US: focal or marked diffuse cortical thickening, hilar fat effacement, or decreased longitudinal to transverse ratio of the node. If no suspicious nodes were found, the largest node on the same side of the primary lesion was included in the study.

Examined nodes were classified by US into three groups blind to the final pathology results:

- Benign/non-specific: ovoid with central fatty hilum and uniform cortical thickness $\leq 3 \mathrm{~mm}$.

- Malignant/infiltrated: either a node with lost hilum or a node with focal/diffuse cortical thickening $>3$ $\mathrm{mm}$ with eccentric hilum (peripheral).

- Indeterminate: node showing cortical diffuse thickening $>3 \mathrm{~mm}$ with central hilum.

\section{The technique of MRI and DWI}

For MRI and diffusion-weighted imaging (DWI), images were acquired using Philips Intera or Achieva machines (1.5 Tesla) followed by post-processing.

For MRI acquisition, three-plane, fast single-shot localizers were initially obtained to localize and plan the sequences. Axial T1-weighted $(\mathrm{TR}=537 \mathrm{~ms}$, TE $=10 \mathrm{~ms})$ and $\mathrm{T} 2$-weighted $(\mathrm{TR}=4123 \mathrm{~ms}, \mathrm{TE}=120 \mathrm{~ms})$ fast spin-echo (FSE) sequences were taken (the number of excitations: 2; the direction of frequency encoding: right to left $(\mathrm{R} / \mathrm{L})$; section thickness: $3 \mathrm{~mm}$; gap: $0.5 \mathrm{~mm}$; field 
of view (FOV): $36-40 \mathrm{~cm}$; matrix: $288 \times 224)$. Short tau inversion recovery (STIR)-weighted images were also acquired $(\mathrm{TR}=4007 \mathrm{~ms}, \mathrm{TE}=70 \mathrm{~ms})$ in the axial and sagittal planes (TR/TE, $1.6 \mathrm{~ms} / 20 \mathrm{~ms}$; number of excitations, 3; direction of frequency encoding: R/L; section thickness, $3 \mathrm{~mm}$; gap, $1 \mathrm{~mm}$; FOV, $36-40 \mathrm{~cm}$; matrix, $288 \times 224$ ).

DWI was acquired in a transverse plane using singleshot echo-planar imaging with suppression of fat, using four $b$ values; low $\left(0-50 \mathrm{~s} / \mathrm{mm}^{2}\right)$, intermediate (200 and $\left.400 \mathrm{~s} / \mathrm{mm}^{2}\right)$, and high $b$ value $\left(1000 \mathrm{~s} / \mathrm{mm}^{2}\right)$ in an attempt to get more accurate values of ADC, using the following parameters: $5000 \mathrm{~ms} / 75 \mathrm{~ms}$ (TR/TE), slice thickness 3-9 $\mathrm{mm}$ with an interslice gap of $0-1.5 \mathrm{~mm}$, and the number of excitations ranging from 1 to 10, 256 $\times 256$ (matrix), $30 \times 30 \mathrm{~cm}$ (FOV).

\section{Qualitative assessment}

Signal intensity in the DWI and the corresponding ADC map images were visually evaluated at intermediate and high $b$ values. Diffusion restriction was indicated by intermediate or high SI on DWI that dropped on the corresponding ADC map.

\section{Quantitative assessment}

Mean ADC values were calculated by manually drawing regions of interest (ROI) in the most hypo-intense (most restricted) portion of the selected node on the ADC map so that the ROI is slightly smaller than the real node to avoid partial volume averaging. Multiple measurements were acquired to calculate mean ADC values. ADC maps were calculated by the MRI system via linear regression analysis of the natural log of signal intensity using all measured $b$ values.

While going through the study, we were faced with inadequate axillary visualization in some cases using the standard breast MRI coil. This may be attributed to the uncomfortable prone positioning with breast coils especially with patient obesity resulting in noticeable motion artifact.

As a result, we tried image acquisition using a chest coil in the supine position with the upper limbs abducted. This allowed a wider FOV and better spatial resolution, resulting in better nodal imaging using FSE techniques due to high contrast, excellent suppression of fat, and relative motion insensitivity during acquisition.

US and DWI-MRI images were conjointly interpreted by two consultant radiologists, having more than 10 years of breast imaging experience. Finally, results of US and DW-MRI were correlated to those of final pathology which served as a standard of reference. All cases with suspicious axillary nodes underwent image-guided sampling. All those with no suspicious axillary nodes were subjected to intraoperative sentinel nodal sampling. All image-guided sampling from suspicious nodes was from level I axillary nodes. Although some patients had additional level II (17 cases) or level III (9 cases) nodal involvement, there were no skip lymphatic lesions and therefore level I nodes were sampled.

For statistical analysis, we used the Statistical Package for the Social Sciences (SPSS, version 25) to code the data which was summarized as mean, standard deviation (SD), median, minimum, and maximum for quantitative data and as frequency (count) and relative frequency (percentage) for categorical data. Quantitative variables were compared using the Mann-Whitney non-parametric test while categorical data was compared using the Chi-square $\left(x^{2}\right)$ test. However, when the expected frequency was less than 5, Exact test was used. Standard diagnostic indices such as sensitivity, specificity, positive predictive value (PPV), negative predictive value (NPV), and diagnostic efficacy were calculated. Receiver operating characteristic curve (ROC) was constructed with the area under curve (AUC) analysis performed to determine best cutoff values of different parameters that detect infiltration. To predict infiltration by combining US and MRI, logistic regression was done, considering $P$ values less than 0.05 as statistically significant.

\section{Results}

This prospective study included 77 axillary nodes from the ipsilateral sides of suspicious breast lesions from 77 patients. These nodes were evaluated by both conventional US and DW-MRI in an attempt to evaluate the added role of DW-MRI in axillary evaluation. The final pathologic diagnosis, which served as our standard of reference, proved that 47 out of the examined 77 nodes (61\%) were infiltrated and 30/77 (39\%) were not infiltrated.

\section{US evaluation of axillary nodes}

Axillary nodes were classified by US into three groups, namely the non-specific (benign), malignant, and indeterminate groups.

\section{US axillary nodal evaluation according to nodal shape}

Considering an ovoid shape as a benign descriptor and a non-ovoid shape as a malignant one, 45 out of the examined 77 nodes (58.4\%) were ovoid and 32 (41.6\%) were non-ovoid resulting in a calculated sensitivity, specificity, PPV, NPV, and accuracy of $61.7 \%$, $90 \%, 90.6 \%, 60 \%$, and $72.7 \%$, respectively, having a significant $P$ value of 0.009 (Table 1 ). 
Table 1 Correlation of nodal shape by US to the final pathology

\begin{tabular}{|c|c|c|c|c|c|c|}
\hline & & Patholc & & & & $P$ \\
\hline & & Infiltra & & Not inf & & \\
\hline & & Count & $\%$ & Count & $\%$ & \\
\hline Lymph node shape & Non-ovoid & 29 & $61.7 \%$ & 3 & $10.0 \%$ & $<0.001$ \\
\hline & Ovoid & 18 & $38.3 \%$ & 27 & $90.0 \%$ & \\
\hline
\end{tabular}

\section{US axillary nodal evaluation according to nodal size}

Axillary nodes were classified according to their size by US in long axis, short axis, and long/short axis ratio (Table 2).

The cut-off value for the nodal short axis (as calculated by ROC curve analysis) between benign and malignant nodes was $0.95 \mathrm{~cm}$. For nodal long axis, we found no cut-off value as malignant and benign results overlapped and consequently, no statistical significance was found for the long axis in differentiating malignant from benign nodes (Fig. 1). For nodal long/short axis ratio, the calculated cut-off value between benign and malignant nodes was 1.9 (Table 3, Fig. 2).

\section{US axillary nodal evaluation according to nodal hilum}

Considering a preserved hilum as a benign descriptor and a non-preserved one as a malignant descriptor, the calculated sensitivity, specificity, PPV, NPV, and accuracy were $72.3 \%, 100 \%, 100 \%, 69.7 \%$, and $83.1 \%$, respectively (Table 4).

US axillary nodal evaluation according to cortical thickness Nodes were classified according to their cortical thickness, where the calculated cut-off value for cortical thickness differentiating infiltrated and non-infiltrated nodes was $5 \mathrm{~mm}$ (as calculated by ROC curve analysis) (Fig. 3). For the pathologically proven infiltrated axillary nodes, the mean cortical thickness was 0.95 , the SD was 0.61 , the median was 0.7 , the minimum was 0.36 , and the maximum was 2.6. On the other hand, for the pathologically proven, non-infiltrated axillary nodes, the mean cortical thickness was 0.39 , the standard deviation was 0.11 , the median was 0.4 , the minimum was 0.2 , and the maximum was 0.6. Accordingly, the calculated sensitivity, specificity, PPV, NPV, and accuracy were 76.6\%,
93.3\%, 94.7\%, 71.7\%, and $83.1 \%$, respectively with $P$ value $<0.001$.

\section{Axillary nodal evaluation according to the studied combined US criteria}

According to the combined studied US criteria (nodal shape, size, hilum, and cortical thickness), 11 out of the examined 77 nodes (14.3\%) were reported by US as benign, 35 (45.5\%) as malignant, and 31 (40.3\%) as indeterminate.

Indeterminate nodes were added to the consideredpositive ones, and all counted up as suspicious, resulting in a total of 66 out of the examined 77 nodes being considered by US as suspicious, out of which 19 were proved benign by pathology (false positive) and 47 were proved malignant (true positive). On the other hand, 11/ 77 nodes were diagnosed as benign by US and confirmed truly benign by pathology (true negative). There were no false negative results concluding a calculated sensitivity, specificity, accuracy, PPV, and NPV for US of $100 \%$, $36.6 \%, 75.3 \%, 71.2 \%$, and $100 \%$, respectively (Table 5).

As for the US-reported indeterminate nodes (31 nodes), 19 turned out to be benign by pathology (false positive) and 12 were confirmed malignant (true positive).

\section{DW-MRI evaluation of axillary nodes Qualitative DW-MRI evaluation of axillary nodes (restricted or not)}

Classification of nodes according to being restricted or not on DW-MRI and correlation with final pathology results was done, considering DWI restriction as a malignant descriptor and non-restriction as a benign one (Table 6). Accordingly, the calculated sensitivity, specificity, PPV, NPV, and accuracy were 76.6\%, 63.3\%, 76.6\%, $63.3 \%$, and $71.4 \%$ respectively with $P$ value $<0.001$.

Table 2 Axillary nodal classification by size according to US

\begin{tabular}{|c|c|c|c|c|c|c|c|c|c|c|c|}
\hline & \multicolumn{10}{|c|}{ Pathology } & \multirow{3}{*}{$\begin{array}{l}P \\
\text { value }\end{array}$} \\
\hline & \multicolumn{5}{|c|}{ Infiltrated } & \multicolumn{5}{|c|}{ Not infiltrated } & \\
\hline & Mean & SD & Median & Minimum & Maximum & Mean & SD & Median & Minimum & Maximum & \\
\hline Size (long axis) & 2.21 & 1.07 & 1.80 & 1.00 & 5.00 & 1.77 & 0.39 & 1.80 & 1.00 & 2.60 & 0.323 \\
\hline Size (short axis) & 1.25 & 0.61 & 1.00 & 0.60 & 3.00 & 0.84 & 0.17 & 0.80 & 0.50 & 1.20 & 0.001 \\
\hline Long/short axis ratio & 1.80 & 0.43 & 1.80 & 1.08 & 2.70 & 2.13 & 0.34 & 2.00 & 1.50 & 2.80 & 0.001 \\
\hline
\end{tabular}




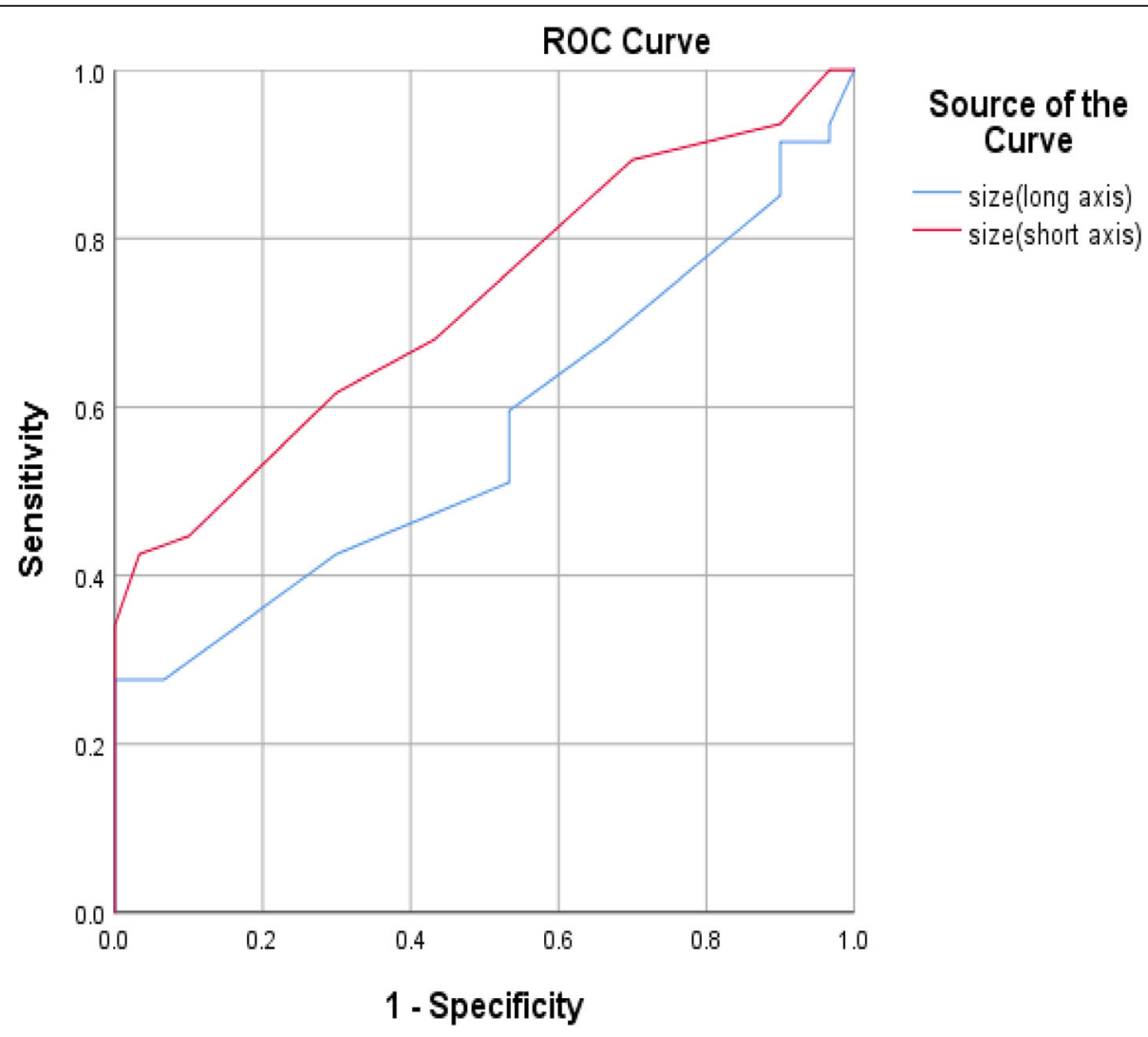

Fig. 1 ROC curve for detection of nodal infiltration using size in long and short axes

\section{$A D C$ evaluation of axillary nodes}

The calculated cut-off value differentiating infiltrated and non-infiltrated nodes for ADC was 0.95 $\times 10^{-3} \mathrm{~mm}^{2} / \mathrm{s}$ (according to ROC curve analysis, AUC $=0.754)$. Accordingly, the calculated sensitivity, specificity, PPV, NPV, and accuracy were $76.6 \%, 63.3 \%, 76.6 \%, 63.3 \%$, and $71.4 \%$, respectively with $P$ value $<0.001$ (Fig. 4). For the pathologyproven, infiltrated axillary nodes, the mean ADC value was 0.84 , the $\mathrm{SD}$ was 0.28 , the median was 0.7 , the minimum was 0.5 , and the maximum was 1.6. For the pathology-proven non-infiltrated nodes, the mean ADC value was 1.12, the SD was 0.32 , the median was 1.1 , the minimum was 0.6 , and the maximum was 1.6.
Performance of DW-MRI in the evaluation of the USdiagnosed indeterminate axillary nodes

US-diagnosed indeterminate nodes were evaluated by DWMRI and classified as either benign or malignant. Out of the 31 US-diagnosed indeterminate nodes, 15 were reported by DW-MRI as malignant, out of which 7 turned out to be benign by pathology (false positive) and 8 were proved malignant (true positive). On the other hand, 16/31 nodes were reported by DW-MRI as benign, out of which 12 were confirmed by pathology to be benign (true negative) and 4 turned out to be malignant (false negative), resulting in a calculated sensitivity of $66.6 \%$, a specificity of $63.1 \%$, and a total accuracy of $64.5 \%$. The PPV and NPV were $53.3 \%$, and $75 \%$, respectively, for DW-MRI evaluation of US-diagnosed indeterminate nodes (Table 7) (Figs. 5, 6, 7, and 8).

Table 3 Diagnostic indices for nodal short and long axial measurements by US

\begin{tabular}{|c|c|c|c|c|c|c|c|c|c|c|}
\hline & \multirow{2}{*}{$\begin{array}{l}\text { Area } \\
\text { under } \\
\text { the } \\
\text { curve }\end{array}$} & \multirow{2}{*}{$\begin{array}{l}P \\
\text { value }\end{array}$} & \multicolumn{2}{|c|}{$95 \%$ confidence interval } & \multirow{2}{*}{$\begin{array}{l}\text { Cut } \\
\text { off }\end{array}$} & \multirow{2}{*}{$\begin{array}{l}\text { Sensitivity } \\
\%\end{array}$} & \multirow{2}{*}{$\begin{array}{l}\text { Specificity } \\
\%\end{array}$} & \multirow{2}{*}{$\begin{array}{l}\text { PPV } \\
\%\end{array}$} & \multirow{2}{*}{$\begin{array}{l}\text { NPV } \\
\%\end{array}$} & \multirow{2}{*}{$\begin{array}{l}\text { Accuracy } \\
\%\end{array}$} \\
\hline & & & Lower bound & Upper bound & & & & & & \\
\hline Size (long axis) & 0.567 & 0.326 & 0.440 & 0.694 & - & - & - & - & - & - \\
\hline Size (short axis) & 0.726 & 0.001 & 0.615 & 0.836 & 0.95 & 61.7 & 70 & 76.32 & 53.85 & 64.94 \\
\hline Long/short axis ratio & 0.726 & 0.001 & 0.613 & 0.840 & 1.0 & 61.7 & 86.7 & 87.88 & 59.09 & 71.43 \\
\hline
\end{tabular}




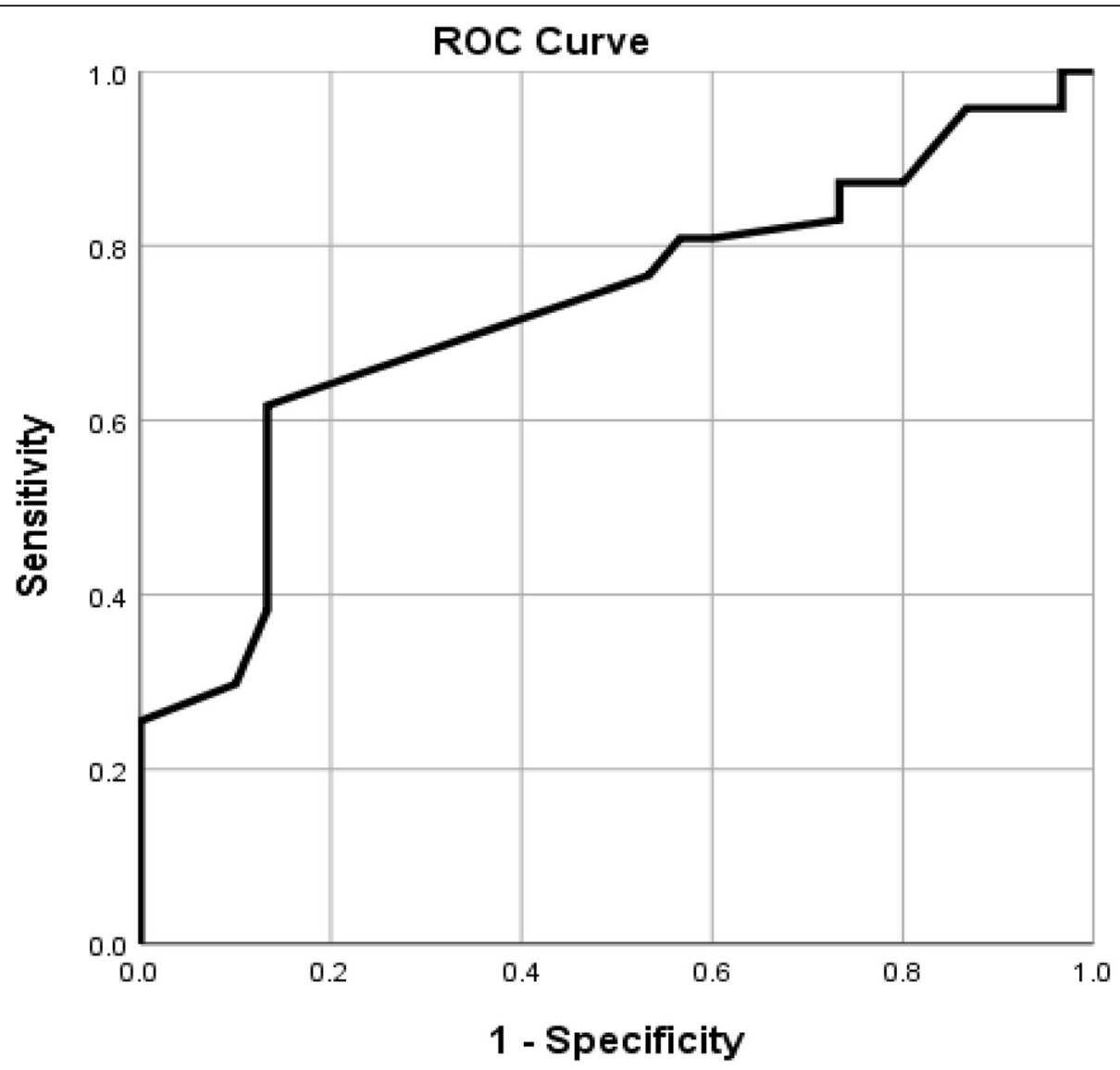

Fig. 2 ROC curve for detection of nodal infiltration using long/short ratio

Performance of DW-MRI in combination with conventional US in axillary nodal evaluation

Using DW-MRI in combination with conventional US improved the overall accuracy of axillary nodal evaluation, having a sensitivity, specificity, PPV, NPV, and accuracy of $76.60 \%, 76.67 \%, 83.72 \%, 67.65 \%$, and $76.62 \%$. So, adding DW-MRI could improve specificity (36.6\%) and accuracy $(75.3 \%)$ of US both to $76.6 \%$.

\section{Discussion}

Axillary nodal metastasis is a cornerstone for the prognosis of cases with breast cancer, affecting their survival rate [6]. However, a definite diagnosis of axillary nodal affection needs tissue biopsy or surgical dissection which are rather invasive procedures and carry considerable

Table 4 Correlation of nodal hilum by US to the final pathology

\begin{tabular}{|c|c|c|c|c|c|c|}
\hline & & \multicolumn{4}{|c|}{ Pathology } & \multirow{3}{*}{$\begin{array}{l}P \\
\text { value }\end{array}$} \\
\hline & & \multicolumn{2}{|c|}{ Infiltrated } & \multicolumn{2}{|c|}{ Not infiltrated } & \\
\hline & & Count & $\%$ & Count & $\%$ & \\
\hline \multirow[t]{2}{*}{ Hilum } & Not preserved & 34 & $72.3 \%$ & 0 & $0.0 \%$ & $<0.001$ \\
\hline & preserved & 13 & $27.7 \%$ & 30 & $100.0 \%$ & \\
\hline
\end{tabular}

complication risks [7]. Therefore, a need has developed for accurate, non-invasive, preoperative axillary imaging in an attempt to reduce complication risks and cut off costs by avoiding unnecessary sampling [8].

In the current study, we examined 77 axillary nodes in females with recently discovered malignant breast lesions, aiming at evaluating the role of DW-MRI, in comparison to US, in the assessment of the axillary nodal status in patients with newly discovered breast cancer, as an attempt to preoperatively detect axillary nodal metastasis, and consequently, reduce unnecessary invasive sampling and avoid axillary over or under-staging. US morphologically classified axillary nodes into benign (non-specific), malignant, and indeterminate groups, whereas DW-MRI functionally classified them into benign (non-specific) and malignant groups.

We found no correlation between patient age and axillary nodal affection $(P$ value $=0.95)$ which is in accordance with other studies [9].

To calculate statistical indices for the performance of US, the US-diagnosed indeterminate group of nodes was added to the pathological group, all together forming a suspicious group. This resulted in calculated sensitivity, specificity, PPV, NPV, and accuracy for the combined 


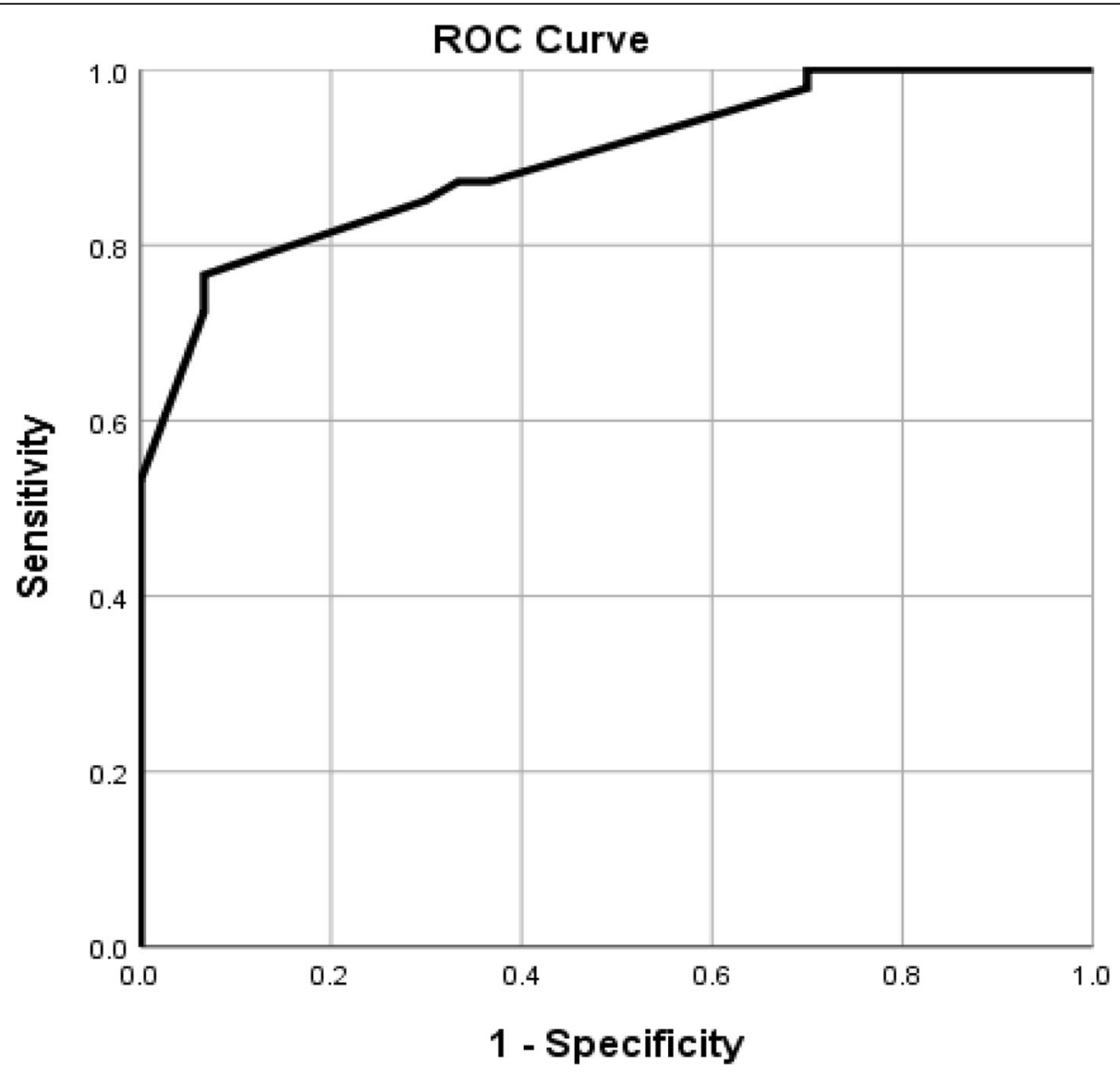

Fig. 3 ROC curve for detection of nodal infiltration using cortical thickness

US criteria of $100 \%, 36.67 \%, 71.21 \%, 100 \%$, and $75.3 \%$, respectively. This may justify the relatively high figure for sensitivity and NPV for US performance where any node having a non-classic feature was added to the suspicious group. On another note, the relatively lower figure for US specificity in our study may be related to the presence of a considerable number of US-diagnosed indeterminate nodes (31/77) (40\%) where, ultimately, many of which turned out to be benign by pathology (19/31) (61\%). US has rather variable reported values for sensitivity (ranging from 49 to $87 \%$ ) and specificity (ranging from 56 to $97 \%$ ) for metastatic axillary nodal detection [10].

Table 5 Correlation of overall US results to those of final pathology

\begin{tabular}{|c|c|c|c|c|c|}
\hline & & \multicolumn{4}{|c|}{ Pathology } \\
\hline & & \multicolumn{2}{|c|}{ Infiltrated } & \multicolumn{2}{|c|}{ Not infiltrated } \\
\hline & & Count & $\%$ & Count & $\%$ \\
\hline \multirow[t]{3}{*}{ US details } & Indeterminate & 12 & $15.5 \%$ & 19 & $24.6 \%$ \\
\hline & Benign & 0 & $0.0 \%$ & 11 & $14.2 \%$ \\
\hline & Malignant & 35 & $45.4 \%$ & 0 & $0.0 \%$ \\
\hline
\end{tabular}

In the current study, cortical thickness proved to be the most sensitive US criterion (76.6\% sensitivity, 93.3\% specificity) for the detection of metastatic axillary nodes as pathologically proven malignant nodes showed significantly higher maximal cortical thickness compared to benign ones, with a calculated cut-off value for cortical thickness that differentiates benign from malignant nodes of $5.2 \mathrm{~mm}$, which is rather comparable to the cutoff value used in previous studies [11].

The results of the current study concluded that the mean short axial diameter of malignant nodes $(1.25 \pm$ $0.61 \mathrm{~cm})$ was significantly higher than that of benign ones $(0.84 \pm 0.17 \mathrm{~cm})(P$ value $=0.001)$. We also found that mean cortical thickness in malignant nodes $(0.95 \pm$ $0.61 \mathrm{~cm})$ was significantly higher than that of benign

Table 6 Distribution of lymph nodes by qualitative DW-MRI

\begin{tabular}{|c|c|c|c|c|c|c|}
\hline & & \multicolumn{4}{|c|}{ Pathology } & \multirow{3}{*}{$\begin{array}{l}P \\
\text { value }\end{array}$} \\
\hline & & \multicolumn{2}{|c|}{ infiltrated } & \multicolumn{2}{|c|}{ not infiltrated } & \\
\hline & & Count & $\%$ & Count & $\%$ & \\
\hline \multirow[t]{2}{*}{ MRI } & Malignant(restricted) & 36 & $76.6 \%$ & 11 & $36.7 \%$ & $<0.001$ \\
\hline & Benign & 11 & $23.4 \%$ & 19 & $63.3 \%$ & \\
\hline
\end{tabular}




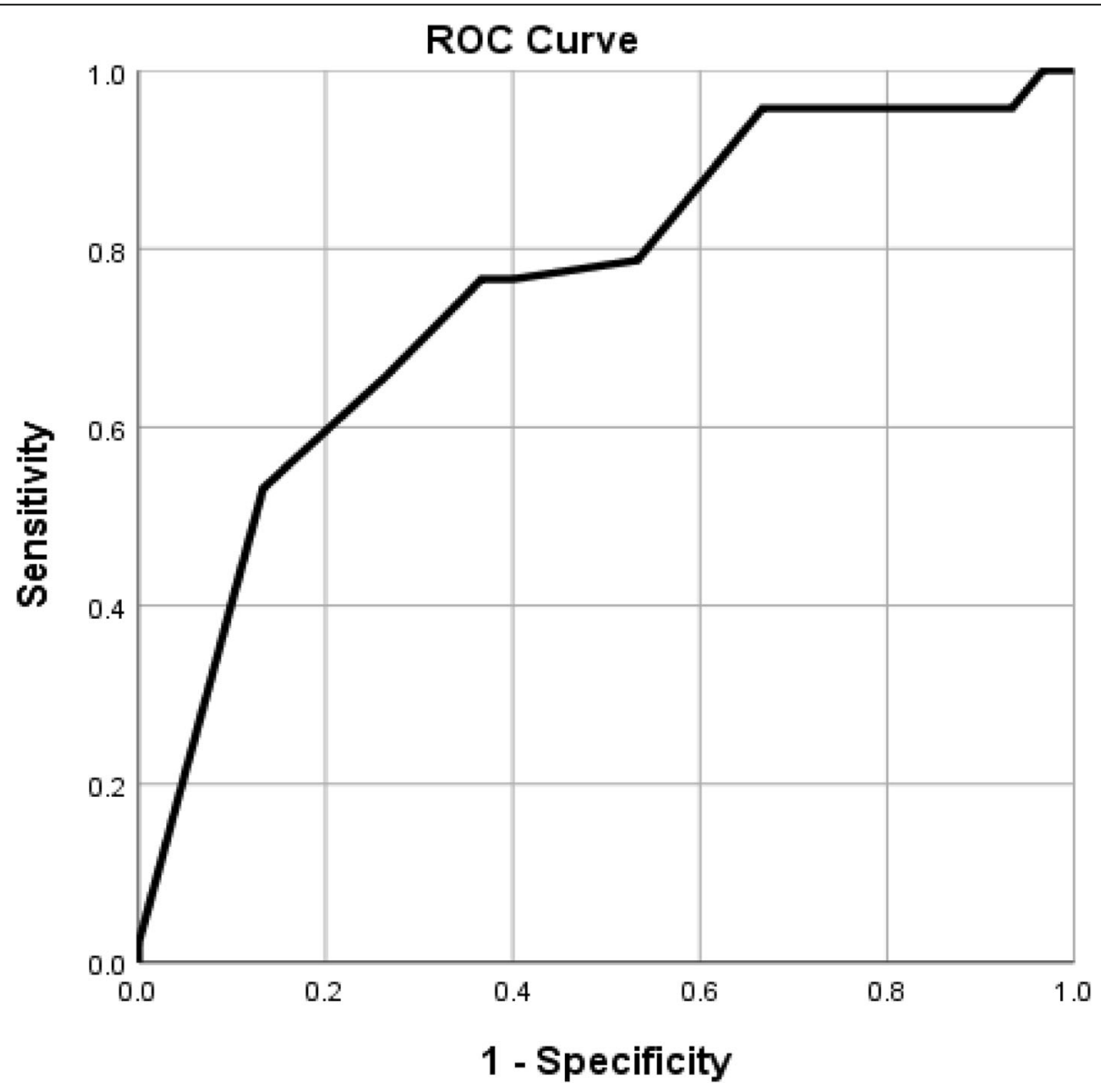

Fig. 4 ROC curve for detection of nodal infiltration using ADC

ones $(0.39 \pm 0.11 \mathrm{~mm})(P$ value $=0.001)$ and that nonpreserved hila were significantly associated with malignant nodes $(P$ value $=0.001)$ which goes in agreement with the results of other previous studies $[3,12]$.

We also concluded that the mean $(\mathrm{L} / \mathrm{T})$ ratio in malignant nodes $(1.8 \pm 0.43 \mathrm{~mm})$ significantly differed from that of benign ones $(2.13 \pm 0.34 \mathrm{~mm})$, having a $P$ value of 0.001 . This agrees with the results of several other previous studies [13, 14]. Yet, other investigators, such as Zaiton and his colleagues [15], reported that they

Table 7 Correlation of DW-MRI classification of US diagnosed indeterminate nodes to the final pathology

\begin{tabular}{|c|c|c|c|c|c|c|}
\hline & & US det & & & & \\
\hline & & Indete & inate & & & \\
\hline & & Pathol & & & & \\
\hline & & Infiltra & & Not in & ated & \\
\hline & & Count & $\%$ & Count & $\%$ & \\
\hline MRI & Malignant & 8 & $66.7 \%$ & 7 & $36.8 \%$ & 0.106 \\
\hline & Benign & 4 & $33.3 \%$ & 12 & $63.2 \%$ & \\
\hline
\end{tabular}

found no statistically significant difference in mean short axial diameter or $\mathrm{L} / \mathrm{T}$ ratio between malignant and benign nodes ( $P$ value: 0.87 and 0.82 , respectively) which also agrees with the results of some other studies [3, 12].

We also concluded the statistically significant value $(P$ value $<0.001$ ) of hilar fat loss in cases of malignant axillary nodal involvement, which agrees with the results of other investigators $[15,16]$.

DW-MRI is a functional magnetic resonance imaging technique that needs no administration of contrast [15].

The results of our current study show that diffusion restriction in nodes was significantly associated with malignancy $(P$ value $=0.001)$, having $76.6 \%$ sensitivity, $63.3 \%$ specificity, $71.4 \%$ accuracy, $76.6 \% \mathrm{PPV}$, and $63.3 \%$ NPV. This agrees with the results of some previous researchers, such as Scaranelo [17] and Abdelatif [3], who reported that differentiation of malignant and benign axillary nodes by visually detecting signal restriction on DWI and ADC maps showed a sensitivity of $84 \%$ and $100 \%$, respectively, a specificity of $77 \%$ and $66.7 \%$, respectively and an accuracy of $80 \%$ and $92.7 \%$, respectively. It also goes in agreement with the results of other 

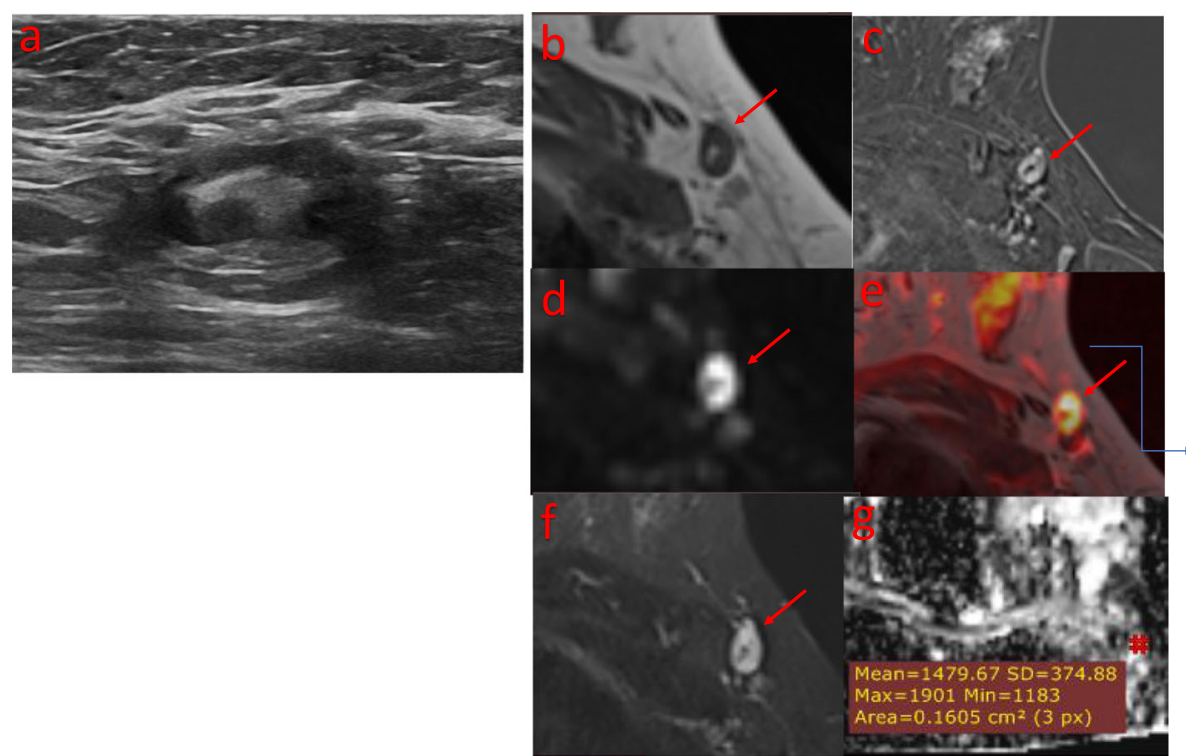

Fig. 5 A 33-year-old female with left Paget's disease and breast lump

previous studies $[15,18,19]$. On the other hand, other researchers did not detect a statistically significant difference comparing DWI signal intensity between inflammatory and metastatic nodes [8].

Quantitative assessment of the diffusibility of the molecules of water in tissues was done via measuring ADC values where the greater the $b$ value, the higher the degree of attenuation of the signal from water molecular motion [20]. This concept was used in some previous studies where rather high $b$ values (1000 and $800 \mathrm{~s} /$ $\mathrm{mm}^{2}$ ) were used, assuming that a high $b$ value could more precisely evaluate the diffusion of water by eliminating capillary perfusion effects $[8,15]$.

We utilized the same principle, where the obtained $A D C$ values were significantly lower for nodes with metastasis than those without metastasis, yet, with an overlap between both groups. Similar results were published by some previous investigators $[8,18,19]$ and an explanation for this overlap was postulated by Wang and his team [8] as they found that malignant nodes were not

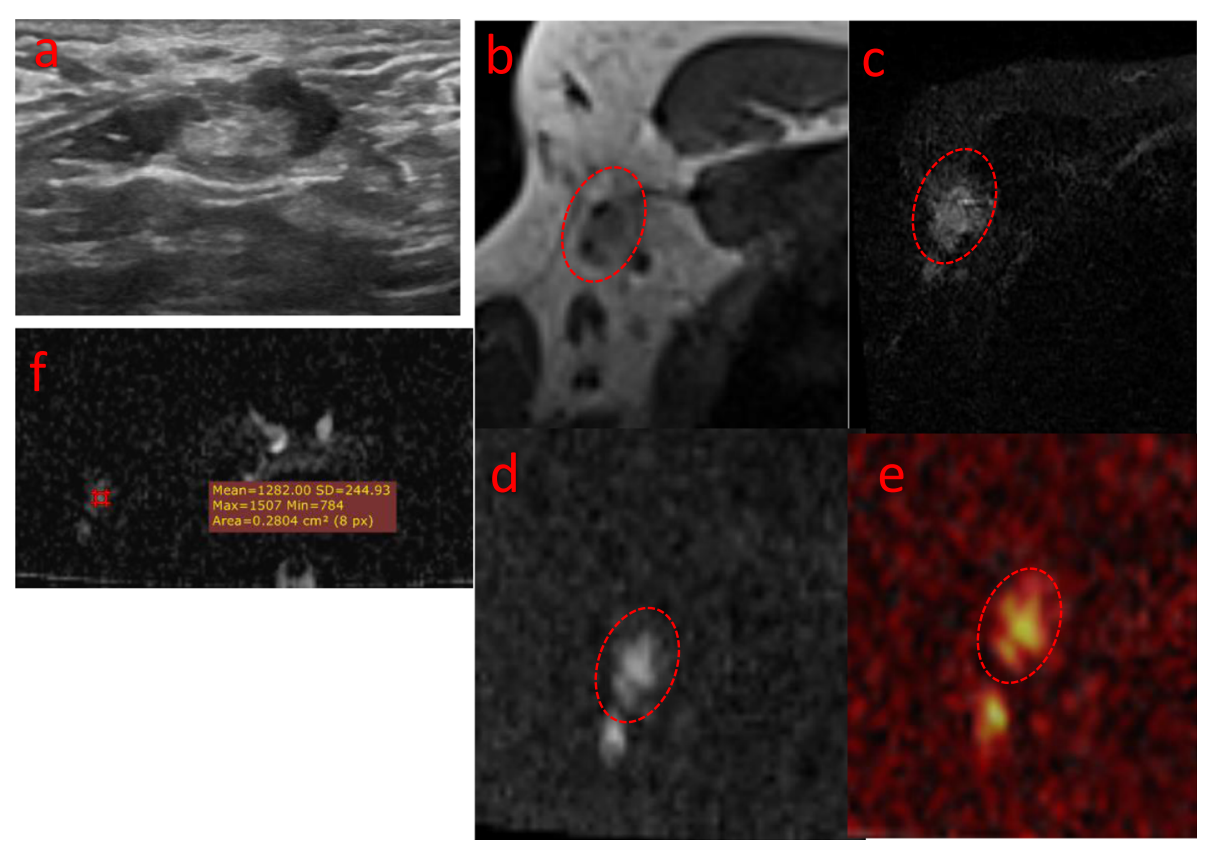

Fig. 6: A 45-year-old female with a right breast lump 


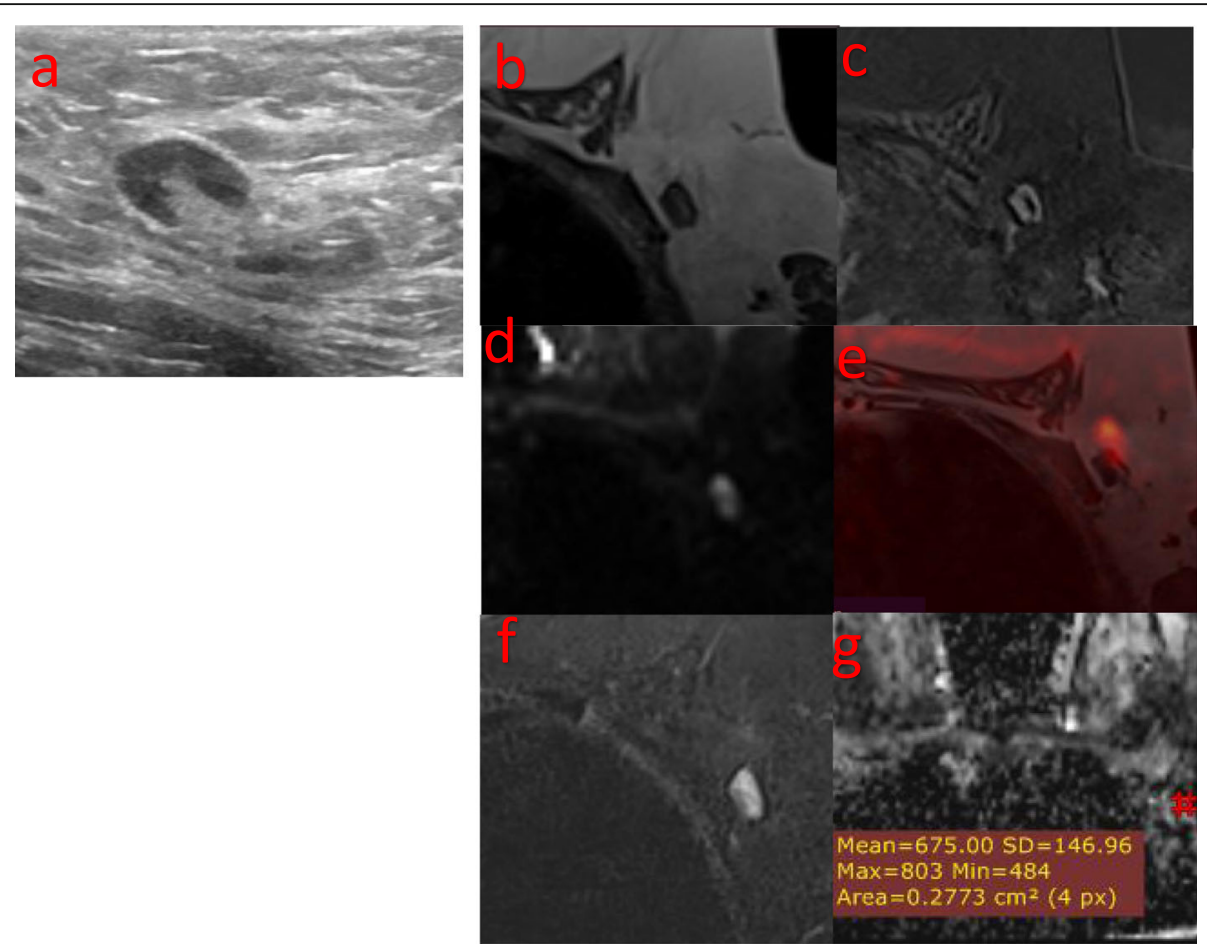

Fig. 7 A 58-year-old lady with a palpable left breast lump
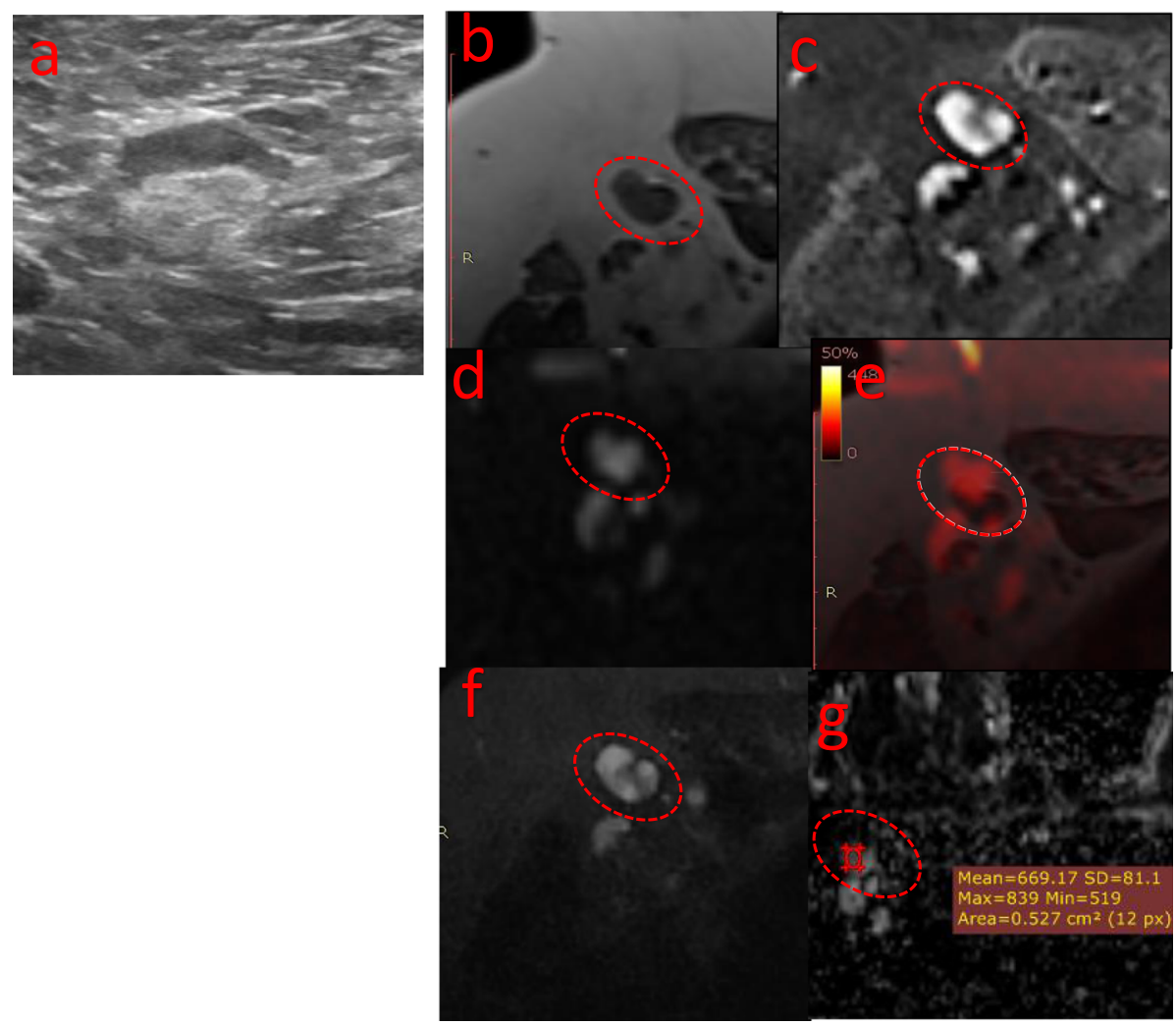

Fig. 8 A 52-year-old female with a palpable right breast lump 
entirely replaced by malignant cells, yielding metastatic areas with lower ADC values and other areas without metastasis, having higher ADC values where a ROI of ADC can be heterogeneous, containing both metastatic and non-metastatic parts. On the other hand, nonmetastatic nodes may show limited diffusibility of molecules of water (and consequently relatively lower ADC values) due to other associated conditions, such as infiltration by inflammatory cells or reactive hyperplasia.

There is a wide variation regarding both the mean and cutoff values of ADC between various studies which may be attributed to many factors such as MRI acquisition parameters [21].

In our current study, we found that the mean ADC values were generally lower for metastatic than for nonmetastatic nodes, which goes in accordance with the results of various other previous studies, all showing rather comparable statistical indices [3, 15, 22-24]. Nevertheless, other studies reported that values of ADC for metastatic nodes were significantly higher than those for non-metastatic ones [25].

Comparing the results of the overall performance of US and DW-MRI in the current study concerning indeterminate nodes, which are those of most clinical concern, we found that DW-MRI had higher specificity (63.1\%) than US (36.6\%) while US had higher sensitivity (100\%).

\section{Limitations}

One of the limiting factors of our current study was only including the most suspicious node, assuming that it correlated to the final histopathological results.

Another limiting point was being confronted by the suboptimal axillary visualization on using the standard breast MRI coil, possibly due to uncomfortable positioning and limitations related to patient obesity. On the other hand, on using a chest coil, we had patients in a more comfortable supine position with abducted arms and overcame the constraints of patient built. This gave way to better axillary imaging due to better spatial resolution and wider FOV using FSE technique which overcomes the problem of motion sensitivity.

One other limitation of the current study was adding the US-diagnosed pathological and indeterminate groups together, forming a suspicious group of nodes so that any node having non-classic US features was included in the suspicious group, which may justify the relatively higher figure for US sensitivity and lower specificity.

\section{Conclusion}

Accurate axillary assessment is important for proper management of cases with recently discovered breast cancer. Although US shows rather excellent sensitivity for axillary nodal visualization, yet specificity could be ameliorated by adding DW-MRI to it. This can be used to reduce the diagnosis of indeterminate nodes which eliminates grey zones and better helps tailor management options, avoiding either over or under staging of the axillary nodal status for each patient.

\section{Abbreviation \\ ADC: Apparent diffusion coefficient; ALND: Axillary lymph node dissection; AUC: Area under the curve; BIRADS: Breast Imaging Reporting Database System Score; DWI: Diffusion-weighted imaging; DW-MRI: Diffusion-weighted magnetic resonance imaging; FOV: Field of view; FSE: Fast spin echo; L/T ratio: Longitudinal to transverse axis ratio; MRI: Magnetic resonance imaging; NPV: Negative predictive value; PPV: Positive predictive value; R/L: Right to left; ROC curve: Receiver operating characteristic curve; ROI: Region of interest; SD: Standard deviation; SM: Sonomammography; SPSS: Statistical Package for the Social Sciences; STIR: Short tau inversion recovery; TE: Time to echo; TR: Time to repetition; US: Ultrasound}

\section{Acknowledgements \\ No acknowledgements.}

\section{Authors' contributions}

DE wrote the manuscript. EB collected patient data. RA worked on image processing and collection of patient images. RK participated in the design of the study and performed the statistical analysis. EB and DE conceived the study, and participated in its design and coordination and helped to draft the manuscript. RK was responsible of revision of the draft from clinical point of view. EK collected the clinical and pathological data. All authors have read and approved the manuscript.

Funding

No funding sources.

Availability of data and materials

The data sets used and analyzed during the current study are available from the corresponding author on reasonable request.

\section{Declarations}

\section{Ethics approval and consent to participate}

The study was approved by the ethical committee of "Faculty of medicine, Cairo University" with ethical committee approval number and date not available. An informed written consent was taken from all subjects.

\section{Consent for publication}

All patients included in this research gave written informed consent to publish the data contained within this study.

\section{Competing interests}

The authors declare that they have no competing interests.

\section{Author details}

${ }^{1}$ Department of Diagnostic and Interventional Radiology, Faculty of Medicine, Cairo University, Cairo, Egypt. ${ }^{2}$ Department of Diagnostic and Interventional Radiology, Baheya Foundation, Cairo, Egypt. ${ }^{3}$ Department of General Surgery, Faculty of Medicine, Cairo University, Cairo, Egypt.

Received: 13 January 2021 Accepted: 29 March 2021

Published online: 07 April 2021

\section{References}

1. Xu Y, Bai X, Chen Y, Jiang L, Hu B, Yu L (2018) Application of real-time elastography ultrasound in the diagnosis of axillary lymph node metastasis in breast cancer patients. Sci Rep 8(1):10234. https://doi.org/10.1038/s41598018-28474-y

2. Van Nijnatten TJA, Ploumen EH, Schipper RJ, Goorts B, Andriessen EH, Vanwetswinkel S, Schavemaker M, Nelemans P, De Vries B, Beets-Tan RGH, Smidt ML, Lobbes MBI (2016) Routine use of standard breast MRI compared to axillary ultrasound for differentiating between no, limited and advanced 
axillary nodal disease in newly diagnosed breast cancer patients. Eur J Radiol 85(12):2288-2294. https://doi.org/10.1016/j.jrad.2016.10.030

3. AbdelLatif M, Shady M, Hegazy MA, Abdo YM (2016) B-mode ultrasound, sono-elastography and diffusion-weighted MRI in differentiation of enlarged axillary lymph nodes in patients with malignant breast disease. EJRNM 47(3): 1137-1149. https://doi.org/10.1016/j.ejrnm.2016.05.018

4. Abdel Razek AA, Abdalla A, Barakat T, El-Taher H, Ali K (2018) Assessment of the liver and spleen in children with Gaucher disease type I with diffusionweighted MR imaging. Blood Cells Mol Dis 68:139-142. https://doi.org/10.1 016/j.bcmd.2016.12.004

5. Foti PV, Ognibene N, Spadola S, Caltabiano R, Farina R, Palmucci S, Milone P, Ettorre GC (2016) Non-neoplastic diseases of the fallopian tube: MR imaging with emphasis on diffusion-weighted imaging. Insights Imaging 7(3):311327. https://doi.org/10.1007/s13244-016-0484-7

6. Li C, Meng S, Yang X, Wang J, Hu J (2014) The Value of T2* in differentiating metastatic from benign axillary lymph nodes in patients with breast cancer-a preliminary in vivo study. PLoS One 9(1):e84038. https://doi.org/1 0.1371/journal.pone.0084038 eCollection 2014

7. He N, Xie C, Wei W, Pan C, Wang W, Lv N, Wu P (2012) A new, preoperative, MRI-based scoring system for diagnosing malignant axillary lymph nodes in women evaluated for breast cancer'. EJR 81(10):2602-2612. https://doi.org/1 0.1016/j.ejrad.2012.03.019

8. Wang J, Liao Q, Zhang Y, Yu C, Bai R, Sun H (2012) Differential diagnosis of axillary inflammatory and metastatic lymph nodes in rabbit models by using diffusion-weighted imaging: Compared with conventional magnetic resonance imaging. Korean J Radiol. 13(4):458-466. https://doi.org/10.3348/ kjr.2012.13.4.458

9. Ashturkar AV, Pathak GS, Deshmukh SD, Pandave HT (2011) Factors predicting the axillary lymph node metastasis in breast cancer: is axillary node clearance indicated in every breast cancer patient? Indian J Surg 73(5): 331-335. https://doi.org/10.1007/s12262-011-0315-5

10. Abe H, Schacht D, Kulkarni K, Shimauchi A (2013) Accuracy of axillary lymph node staging in breast cancer patients. An observer-performance study comparison of MRI and ultrasound. Acad Radiol 20(11):1399-1404. https:// doi.org/10.1016/j.acra.2013.08.003

11. Rotim T, Kristek B, Turk T, Kretić D (2017) Measurable and unmeasurable features of ultrasound lymph node images in detection of malignant infiltration. Acta Clin Croat 56(3):415-424. https://doi.org/10.20471/acc.2017. 56.03 .08

12. Choi JJ, Kang BJ, Kim SH, Lee JH, Jeong SH, Yim HW, Song BJ, Jung SS (2011) Role of sonographic elastography in the differential diagnosis of axillary lymph nodes in breast cancer. JUM 30(4):429-436. https://doi.org/10. 7863/jum.2011.30.4.429

13. Gupta A, Rahman K, Shahid M, Kumar A, Qaseem SM, Hassan SA, Siddiqui FA (2011) Sonographic assessment of cervical lymphadenopathy: Role of high-resolution and color Doppler imaging. Head Neck 33(3):297-302. https://doi.org/10.1002/hed.21448

14. Ragheb AS, Abdel Rahman HM, Ismail AA, Nawar N (2014) Can diffusion weighted image and apparent diffusion coefficient (ADC) differentiate benign from malignant cervical adenopathy? EJRNM 45(2):377-386. https:// doi.org/10.1016/j.jirnm.2014.01.016

15. Zaiton F, Shehata SM, Abo Warda MH, Alekrashy MA (2016) Diagnostic value of MRI for predicting axillary lymph nodes metastasis in newly diagnosed breast cancer patients: diffusion-weighted MRI. EJRNM 47(2):659-667. https://doi.org/10.1016/j.ejrnm.2016.03.008

16. Kaur N, Sharma P, Garg A, Tandon A (2013) Accuracy of individual descriptors and grading of nodal involvement by axillary ultrasound in patients of breast cancer. Int J Breast Cancer 2013:930596-930596. https:/ doi.org/10.1155/2013/930596

17. Scaranelo AM, Eiada R, Jacks LM, Kulkarni SR, Crystal P (2012) Accuracy of unenhanced MR imaging in the detection of axillary lymph node metastasis: study of reproducibility and reliability. Radiol. 262(2):425-434. https://doi.org/10.1148/radiol.11110639

18. Perrone A, Guerrisi P, Izzo L, D’Angeli I, Sassi S, Lo Mele L, Marini M, Mazza D, Marini M (2014) Diffusion-weighted MRI in cervical lymph nodes: differentiation between benign and malignant lesions. Eur J Radiol 77(2): 281-286. https://doi.org/10.1016/j.ejrad.2009.07.039

19. Ismail AA, Hasan DI, Abd-Alshakor H (2014) Diagnostic accuracy of apparent diffusion coefficient value in differentiating metastatic form benign axillary lymph nodes in cancer breast. EJRNM 45(3):1011-1016. https://doi.org/10.1 016/j.ejrnm.2014.06.003
20. Kim JK, Jang YJ, Cho G (2009) Multidisciplinary functional MR imaging for prostate cancer. Korean J Radiol 10(6):535-551. https://doi.org/10.3348/kjr.2 009.10.6.535

21. Zhang F, Zhu L, Huang X, Niu G, Chen X (2013) Differentiation of reactive and tumor metastatic lymph nodes with diffusion-weighted and SPIOEnhanced MRI. Mol Imaging Biol. 15(1):40-47. https://doi.org/10.1007/s113 07-012-0562-2

22. Fornasa F, Nesoti MV, Bovo C, Bonavina MG (2012) Diffusion-weighted magnetic resonance imaging in the characterization of axillary lymph nodes in patients with breast cancer. JMRI 36(4):858-864. https://doi.org/10.1002/ jmri.23706

23. Chung J, Youk JH, Kim JA, Gweon HM, Kim EK, Ryu YH, Son EJ (2014) Role of diffusion-weighted MRl: predicting axillary lymph node metastases in breast cancer. Acta Radiol 55(8):909-916. https://doi.org/10.1177/02841 85113509094

24. Abdel Razek AA, Abdel Lattif M, Denewer A, Farouk O, Nada N (2016) Assessment of axillary lymph nodes in patients with breast cancer with diffusion-weighted MR imaging in combination with routine and dynamic contrast MR imaging. Breast Cancer Res 23(3):525-532. https://doi.org/10.1 007/s12282-015-0598-7

25. Kamitani T, Hatakenaka M, Yabuuchi H, Matsuo Y, Fujita N, Jinnouchi M, Nagao M, Shirahane K, Tokunaga E, Honda H (2013) Detection of axillary node metastasis using diffusion-weighted MRI in breast cancer. Clin Imaging 37(1):56-61. https://doi.org/10.1016/..clinimag.2012.02.014

\section{Publisher's Note}

Springer Nature remains neutral with regard to jurisdictional claims in published maps and institutional affiliations.

\section{Submit your manuscript to a SpringerOpen ${ }^{\circ}$ journal and benefit from:}

- Convenient online submission

- Rigorous peer review

- Open access: articles freely available online

High visibility within the field

- Retaining the copyright to your article

Submit your next manuscript at $\boldsymbol{\nabla}$ springeropen.com 